\title{
Article
}

\section{Barriers to the uptake and use of feedback in the context of summative assessment}

Harrison, Christopher, Könings, Karen D., Schuwirth, Lambert, Wass, Valerie and van der Vleuten, Cees

Available at http://clok.uclan.ac.uk/33418/

Harrison, Christopher ORCID: 0000-0002-6008-4483, Könings, Karen D., Schuwirth, Lambert, Wass, Valerie and van der Vleuten, Cees (2014) Barriers to the uptake and use of feedback in the context of summative assessment. Advances in Health Sciences Education, 20 (1). pp. 229-245. ISSN 1382-4996

It is advisable to refer to the publisher's version if you intend to cite from the work. http://dx.doi.org/10.1007/s10459-014-9524-6

For more information about UCLan's research in this area go to http://www.uclan.ac.uk/researchgroups/ and search for <name of research Group>.

For information about Research generally at UCLan please go to http://www.uclan.ac.uk/research/

All outputs in CLoK are protected by Intellectual Property Rights law, including Copyright law. Copyright, IPR and Moral Rights for the works on this site are retained by the individual authors and/or other copyright owners. Terms and conditions for use of this material are defined in the policies page.

\section{CLoK}

Central Lancashire online Knowledge www.clok.uclan.ac.uk

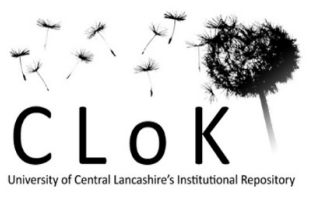




\section{Barriers to the uptake and use of feedback in the context of summative assessment}

\section{Running head:}

\section{Barriers to uptake of feedback}

Christopher J. Harrison

Keele University, School of Medicine, UK

Karen D. Könings

Maastricht University, Department of Educational Development and Research, Faculty of Health, Medicine and Life Sciences, Netherlands

Lambert Schuwirth

Flinders University, School of Medicine, Australia

Valerie Wass

Keele University, School of Medicine, UK

Cees van der Vleuten

Maastricht University, Department of Educational Development and Research, Faculty of Health, Medicine and Life Sciences, Netherlands

Correspondence should be addressed to: Chris Harrison, Keele University Medical School, David Weatherall Building, Keele University, Keele, Staffordshire, ST5 5BG, UK.

Email: c.j.harrison@keele.ac.uk

Tel: +441782 734677; Fax: +441782 734637 


\section{ABSTRACT}

Despite calls for feedback to be incorporated in all assessments, a dichotomy exists between formative and summative assessments. When feedback is provided in a summative context, it is not always used effectively by learners. In this study we explored the reasons for this. We conducted individual interviews with 17 students who had recently received web based feedback following a summative assessment. Constant comparative analysis was conducted for recurring themes. The summative assessment culture, with a focus on avoiding failure, was a dominant and negative influence on the use of feedback. Strong emotions were prevalent throughout the period of assessment and feedback, which reinforced the focus on the need to pass, rather than excel. These affective factors were heightened by interactions with others. The influence of prior learning experiences affected expectations about achievement and the need to use feedback. The summative assessment and subsequent feedback appeared disconnected from future clinical workplace learning. Socio-cultural influences and barriers to feedback need to be understood before attempting to provide feedback after all assessments. A move away from the summative assessment culture may be needed in order to maximise the learning potential of assessments.

\section{Key words:}

Assessment for learning

Feedback

OSCE

Summative assessment

Undergraduate medical education 


\section{INTRODUCTION}

The importance and potential power of feedback is well-established, both in general educational settings and in relation to the health care professions (Hattie and Timperley, 2007; Shute, 2008; van de Ridder et al., 2008; Archer, 2010). As a result, there have been calls to provide feedback after all forms of assessment (Norcini et al., 2011). However, there often remains an artificial dichotomy between formative and summative assessments. In the former, the focus is on using the assessment to enhance learning. By contrast, the focus in summative assessments is on determining if the examinees are competent to progress to the next level. This often limits the effort teachers are willing to put into providing feedback but also limits the uptake of feedback by students especially those that have passed the assessment (Archer, 2010). Though high-stakes assessments have the potential to generate much useful information about the learner, they are often ultimately reduced to a single pass-fail decision. Though this decision-making is clearly important, the lack of feedback provision represents a potential missed opportunity, if it can be achieved without increasing the burden on assessors.

This reductionist approach to assessment has been challenged (Schuwirth and van der Vleuten, 2011). In a high-stakes OSCE assessment, the scores for each item or station are typically added together to make one final mark. Thus, a student who scores full marks on a communication skills OSCE station but zero marks on a resuscitation station will receive the same overall score as one who scores zero marks in communication skills but full marks in resuscitation, even though the information learnt about each learner's competence may be 
vastly different. Similar analogies apply when totalling up marks from written assessments, such as single-best-answer questions.

Attempts to challenge this artificial dichotomy and instead provide feedback after highstakes assessments are not necessarily successful. From the limited evidence, it appears that students will not always take up the offer to receive feedback, or will make limited use of it, following summative written assessments or OSCEs (Sinclair and Cleland, 2007; Harrison et al., 2013). Performance appears to be an influential factor, with students who perform very well, and those who fail the assessment, tending to make more use of the feedback, whereas those students who just passed make least use (Harrison et al., 2013). These studies were quantitative in nature, so cannot explain why students might not engage with feedback in these circumstances. However, if we really want to harness the power of assessment to enhance future learning, we need to understand why learners engage with feedback in a summative context or decide not to. The literature on feedback has been developing rapidly in recent years but rarely considers feedback in a summative context. It is worthwhile considering if any of the conclusions may be applicable to the summative setting.

Although feedback is a highly complex process, there is general agreement that several important steps need to be negotiated in order for it to be successful in improving the knowledge or performance of the learner (Hattie and Timperley, 2007; Shute, 2008; Archer, 2010). Firstly, a learner needs to be receptive to receiving it. Secondly, the learner must 
understand the message being given, so it must be such that it aligns with the learner's frame of reference (Kluger and DeNisi, 1996). Thirdly, the learner needs to set concrete, meaningful and attainable goals, and then take steps to reach them (Hattie and Timperley, 2007; Shute, 2008). The importance of creating a 'culture of feedback' has been advocated (Archer, 2010). Recent work has demonstrated how social and cultural influences within a profession can influence an individual's receptivity and responsiveness to feedback (Watling et al., 2013). These issues will be considered briefly for their potential role in affecting learners' receptivity to feedback after summative assessment.

Feedback-seeking behaviour can be defined as "the conscious devotion of effort towards determining the correctness and adequacy of one's behaviours for attaining valued goals" (Crommelinck and Anseel, 2013). Feedback-seeking behaviour by employees in the workplace has been extensively studied by organisational psychologists. Ashford and Cummings (1983) proposed three main motives for feedback-seeking behaviour. Firstly, there is the need to obtain useful information; secondly there is the need to preserve or enhance one's own ego; and thirdly there is the need to defend or enhance the impression others hold of the person (Ashford and Cummings, 1983). For instance, employees, when they are confident that their performance is good, seek feedback not to obtain information for themselves, but rather to convey or highlight information to others (Morrison and Bies, 1991). There is some empirical support that these findings may be applicable to a medical education setting (Teunissen et al., 2009; Bok et al., 2013). Bok et al. (2013) found that characteristics of the feedback provider, characteristics of the feedback seeker, and the relationship between the feedback seeker and provider are all important. 
In the context of the current study, it may be more relevant to consider why learners may refrain from seeking, or actively avoid, feedback. As well as feedback-seeking behaviour, Moss et al (2003) proposed that active feedback-avoiding behaviour also exists. Poorer performers in the workplace may actively avoid feedback when they are aware that their performance is poor. Other studies have also demonstrated that poorer performers are less likely to seek feedback (Northcraft and Ashford, 1990; Morrison and Bies, 1991). We are not aware of any studies of active feedback-avoiding behaviour in the medical education literature.

Educators need to ensure that students understand the feedback message. Vagueness and uncertainty will lead to a higher cognitive load for learners, which can impair the response to feedback (Shute, 2008). Directive feedback, in which clear guidance is given on what needs to be changed and which strengths should be maintained, appears to be most useful for novice or struggling learners, whereas more facilitative feedback, which comprises comments and suggestions, is acceptable for more experienced learners (Shute, 2008). At the same time, a very long complex feedback message is not necessarily helpful, as learners may lose interest (Shute, 2008).

Even if the feedback message is clearly understood, learners need to be willing to accept the feedback and make use of it. Learners will tend to make little use of feedback if the information provided confirms what they already knew (Hattie and Timperley, 2007). 
Feedback may be disregarded if it is lacking in credibility; for example because the feedback giver has not observed a learner directly (Watling et al., 2013). Learners are apprehensive about receiving feedback information which challenges their own self-assessment of their abilities (Mann et al., 2011). Critical feedback can induce strong emotional reactions which may block effective use (Sargeant et al., 2008). Some learners appear more interested in using feedback as a means of boosting their self-confidence rather than as a device to correct knowledge or skill deficiencies (Eva et al., 2012).

As well as considering the individual learner's response to feedback, Watling et al. (2013) advocate consideration of the social and cultural contexts in which feedback and learning takes place. Socio-cultural theory, which has been used to interpret experiential learning in the clinical workplace, may also help us to gain a greater understanding of the complex learning that occurs, or fails to occur, following summative assessments (Yardley et al., 2012). Different professions appear to have different organisational cultures with respect to feedback, which influence how learners perceive the constructiveness and credibility of feedback they receive (Watling et al., 2013). This challenges conventional approaches to feedback in medical education, which focus primarily on the feedback delivery skills of faculty members (Ramani and Krackov, 2012).

The effects that assessment can have on learning are clearly important. These have previously been categorised as pre-assessment, post-assessment and pure assessment effects (Dochy et al., 2007). Studies to date on the learning effects of summative assessment 
have focussed on the pre-assessment effects and not considered the post-assessment effects (Rudland et al., 2008; Cilliers et al., 2010, 2012; O'Carroll and Fisher, 2013). In these studies, there are complex and multifaceted interactions between various factors in an assessment system which together influence learning, sometimes in a positive way, but often in a negative manner. Students pick up implicit messages from the teaching staff and their fellow students regarding the expectations of the learning that is required. Students' perceptions of their ability to bring about a particular outcome, and the 'costs' involved in reaching a desired outcome, may also influence their learning.

Although the literature on feedback is currently expanding rapidly, there is a lack of research into feedback in the context of summative assessment. This study therefore seeks to fill this gap by building on the theoretical models in the educational and organisational psychology literature. We aimed to explore why students failed to make more use of feedback after summative assessment, and which factors were influential.

\section{METHODS}

\section{Context}

The study took place at Keele University School of Medicine in 2011 with students from the third year (out of five). Two-thirds of the way through the year, students have a summative 12-station Objective Structured Clinical Examination (OSCE), including stations on historytaking, clinical examination and practical procedural skills. Students have to pass at least 8 stations in order to progress to the next year. Each station lasted for 8.5 minutes. New 
stations are introduced each year to minimise the risk of students 'studying to the test'. Students have to pass the OSCE, as well as written assessments, in order to progress to Year 4. Following the OSCE, they have a further 12 weeks of study within the year, based in general practice and in selected clinical areas of their choice. Individualised feedback about each student's performance was released via a website on the same day the results were published but there was no compulsion for students to view the feedback. Students could choose to look at the feedback in different ways; they could view a breakdown of the skills assessed in each station or across the OSCE as a whole. They could also compare their performance with the cohort as a whole. More details about this have been published elsewhere (Harrison et al., 2013). A number of screencaptures are shown in Appendix A. In years 1 and 2, the students had received feedback on summative written and practical skills assessments in large group classes. They had not had a formative OSCE earlier in Year 3, but had received more informal teaching regarding preparation for the assessment.

\section{Participants and Sampling}

Recruitment of students followed a maximum variation sampling approach to ensure that the sample included students with a wide range of achievement in written and OSCE assessments, and who had used the website in very different ways. Maximum variation sampling helps to achieve information-rich cases for in-depth study (Patton, 2002). To preserve anonymity, and in order to avoid any suggestion of coercion, students received an initial email from an administrator, rather than directly from the researchers. Interviewing continued until theoretical saturation was reached. 
Individual, semi-structured, face-to-face interviews were conducted in June and July 2011 by the principal researcher $(\mathrm{CH})$, who had previously had little direct interaction with the students. This method was chosen in order to gain in-depth information and encourage deep exploration of personal matters in a way that is often not possible in a group setting (DiCicco-Bloom and Crabtree, 2006). The interviewing technique followed recognised methods of rapport development and use of exploratory open-ended questions recommended by DiCicco-Bloom and Crabtree (2006). All the interviews were audiorecorded. The interviews lasted 30 - 60 minutes. Questions explored students' experiences of their recent OSCE, their perceptions of the feedback they received and its impact. The interview protocol is shown in Appendix B. Themes which emerged in earlier interviews were explored in subsequent interviews.

In total, data were derived from 17 participants. It proved hard to recruit students who had only just passed the assessment (passed 8 or 9 OSCE stations). As these were a group who had been shown to have made little use of feedback, recruitment continued to focus on those students who had accessed the feedback in only a limited manner but had a slightly higher level of performance (Harrison et al., 2013). Details of the participants are shown in Table 1. Ten (59\%) of the interviewees were female, which was identical to the proportion of females in the year group as a whole. Twelve ( $80 \%$ of those who provided information) had entered directly from school which is comparable to the cohort as a whole (73\%).Participants visited the website on one to five separate occasions, viewing between 
46 to 316 webpages in $6-70$ minutes. These figures were closely comparable to the cohort as a whole.

TABLE 1 TO APPEAR AROUND HERE

\section{Data Analysis}

Verbatim transcriptions of the interviews were analysed with the assistance of computerassisted qualitative data analysis software (NVIVO version 9, QSR International Pty Ltd, Doncaster, Victoria, Australia). Data were analysed using the constant comparative method, which comprises simultaneous coding and analysis of data in order to develop and refine concepts and explore their inter-relationship (Corbin and Strauss, 2008). Analysis combined coding which was guided by a priori awareness of the feedback literature relevant theories with inductive coding which emerged from the data. Findings from the interviews were triangulated, with the participants' consent, with information from each participant's usage of the website, and their performance in the assessments (Lingard et al., 2005). The

principal researcher $(\mathrm{CH})$ coded all transcripts and constructed initial themes. A quarter of the transcripts were reviewed by a second researcher (VW) against the coding framework and themes. A third researcher (SY), unconnected from the main research project, independently coded two transcripts. Discrepancies were resolved by discussion.

\section{Ethical issues}


The study was approved by Keele School of Medicine Ethics Committee. Participants were given an information sheet and asked to sign a consent form before the interview commenced. As a token of thanks for their participation, participants were given a gift voucher, worth f10. They were informed of the availability of the gift voucher at the recruitment stage.

\section{RESULTS}

Five themes emerged from our analysis: (i) the power of the summative assessment culture and its negative impact on the use of feedback; (ii) the influence of strong emotions; (iii) the influence of social interactions with others; (iv) the influence of prior learning experiences on expectations; $(v)$ the disconnection between assessment and future learning. These key themes will be described in more detail and highlighted by representative comments from participants.

\section{Power of summative assessment culture}

Summative assessment created a powerful culture that was dominated by fear of failure and potential punishment, i.e. the need to take re-sits.

I always say that I'm happy to just pass. Because it's a pass and I never ever want to have to go through everything again, to resit.

(Student 8, Female, passed 10 out of 12 stations, quartile rank 3, spent 14 minutes on the website)

I thought l'd failed it. It was horrible, horrible, horrible. 
(Student 9, Female, passed 10 stations, quartile rank 3, spent 70 minutes on the website)

The fear of failure, and the need to jump over the assessment hurdle, pervaded most interviews and prevented the OSCE from being regarded as a learning opportunity, but was instead an extrinsic motivator.

"Everyone's so worried that they're gonna fail that they just work really hard." (Student 3, Female, passed 12 stations, quartile rank 1, spent 6 minutes on the website)

This culture paradoxically focused more attention on students who failed and required the punishment, while ignoring students who passed the assessment:

It's always, you get your mark and, and if you, if you fail then you see someone about it, and if you pass that's it, you, you go off on your own way sort of thing. Even if the person who passed only got a mark more than the person who's failed. (Student 2, Male, passed 12 stations, quartile rank 4, spent 63 minutes on the website)

The attitude towards the hurdle being seen as an endpoint, not as a stepping stone towards future learning, was perceived as being reinforced by the medical school.

“Well I must admit, I think part of the reason why I don't put much thinking into the results might be because, here at the medical school, when the exam's over it's over. They don't mention it, you get on with your normal day-to-day activities." 
(Student 5, Male, passed 10 stations, quartile rank 1, spent 8 minutes on the website)

\section{Influence of strong emotions}

The fear of failure generated very strong emotions in the students. On the day of the OSCE, the wait for the exam to begin was a time of high anxiety, with several participants being physically sick. Fear of failure was dominant while awaiting the results, which led to even greater anxiety. Some students were too fearful to open the results and asked colleagues or friends to do it on their behalf.

The timing of the feedback was a factor. In this instance it was released while they were revising for other written exams and some did not want it to disturb their revision for these exams, as the fear of receiving a failing mark was likened to a grief reaction:

Now, I was in two minds whether to look at it, because, obviously, there was written exams to prepare for.... because I think if I hadn't have done so well, I don't think I would have, er, been in the correct mind frame for my written exams. Because I think whenever you've done bad in exams you need a bit of time to mourn.

(Student 16, Female, passed 11 stations, quartile rank 4, spent 6 minutes on the website)

In contrast, the period after the release of results was marked, in the vast majority of students, by an intense feeling of relief, as the goal of avoiding the re-sits had been achieved. This reduced the motivation of students to engage with the specific feedback. The 
enormous relief at overcoming the assessment hurdle, following the fear of failure, appeared to block a more rational interest in the feedback. The anxiety, both while performing the OSCE, and while awaiting the results, appeared to intensify the feeling of relief.

And I heard that the results were up. And I was really, really nervous. I had to get my friend to check it for me because I was so nervous. And because of the feelings, like I thought I'd failed and I really didn't want to have to retake them again. But when my friend checked it and I was told that I'd passed, I was over the moon.

(Student 8, Female, passed 10 stations, quartile rank 3, spent 14 minutes on the website)

\section{Social interactions with others}

Interactions with clinical teachers, peers, parents and partners (boyfriends/girlfriends) reinforced the need to avoid failure. Students perceived that clinical teachers regarded the OSCE as a hoop to jump through, for which a pass was sufficient, whereas the real clinical learning took place separately, and often after graduation.

Some [teachers] say it's about passing and say that the true, erm, medicine begins when you become a doctor. I've heard that quite a few times from clinicians. Some, erm, push for excellence, but don't push for excellence for academic success, but for excellence for your future practice as a doctor. 
(Student 16, Female, passed 11 stations, quartile rank 4, spent 6 minutes on the website)

There was rarely any in-depth discussion of the feedback between students, other than discussion as to whether they needed to re-sit or not, which reinforced the focus on the summative pass-fail culture rather than on using the feedback for learning. Students perceived a strongly competitive attitude among the cohort, partly due to academic ranking being used in applications for the first jobs when leaving medical school and partly due to a perceived inherent competitiveness from medical students being high academic achievers. This attitude was rarely explicitly discussed between students and they struggled to explain why it existed so strongly. Nevertheless, almost all students sensed the hidden competition and this appeared to influence their response to the feedback. Students often expressed contradictory opinions about their own and others' attitudes to competition:

"I find it useful to see how I am doing in comparison to everyone else....I hate it when everyone comes out and asks 'what percent did you get?....I'd like to see myself, but I don't want everyone else to be comparing'"

(Student 14, Male, passed 11 stations, quartile rank 1, spent 36 minutes on the website)

Students were reluctant to reveal to others that they had performed well for fear that it would be interpreted by others as arrogant or uncaring towards those who had performed badly. Others felt unable to keep up with the academically strongest performers, and 'opted out' of the competition and aimed just to pass. 
I wouldn't say, 'Oh, you know what, I got a good mark,' where some people do, but that's just not me because I just don't like showing off. I don't like people thinking that I think that I'm really good [uh-huh], because there's things that I'm not good at, so I don't wanna be seen as that.

(Student 5, Male, passed 10 stations, quartile rank 1, spent 8 minutes on the website)

Students also had to negotiate a complex situation of competition with their partner without upsetting the relationship.

I wouldn't want to make myself sound like I think I'm better than her. And I wouldn't want her to make out that she's better than me, so it works two ways.'

(Student 5, Male, passed 10 stations, quartile rank 1, spent 8 minutes on the website)

Although students seemed more willing to show the feedback data to parents, this was usually in order to demonstrate that they are performing to a satisfactory level. They valued positive feedback from parents more than from examiners.

Erm, I think, in a way, it was, sort of, like, me showing, 'Oh, look Mum,' like, 'this is the pass rate and I got,' you know, 'three marks higher than the pass rate, that's good, isn't it?' Erm, and that's because I think everyone do seek some, kind of, approval and some congratulations, but that needs to be from someone who knows you on a personal level, and that's why I think I didn't wanna have congratulations from the examiners I didn't know, I wanted it from someone else. 
(Student 5, Male, passed 10 stations, quartile rank 1, spent 8 minutes on the website)

Parents reinforced the attitude among students to be content with just passing the assessment.

My mum's just like, 'As long as you pass it doesn't matter,' so she's, like, I suppose that's where l've got it from [uh-huh], because when I get stressed, she's, like, 'Oh, it doesn't even matter anyway, just as long as you pass, you're fine.'

(Student 14, Male, passed 11 stations, quartile rank 1, spent 36 minutes on the website)

\section{Influence of prior learning experiences on expectations}

The culture in which students had learnt prior to attending medical school affected their expectations about achievement and the need to use feedback to improve. Students reported difficulty in adjusting to a change in their expectations for academic achievement; as school pupils they were used to coming near the top of the class, but now as medical students they were well down the field. This led to a sense of futility at being unable to achieve marks towards the top of the year. As a result, they avoided trying too hard and instead were content with the lower goal of simply passing the assessment. This further reinforced the summative nature of the assessment.

I think that's probably ... because most people at Med School will get As, A*s, things like that and to get that you get like $90 \%$ over and above and marks like that and I think that, I still sometimes compare it in a way. I think that when I did well, in my A- 
level I you know I got ... I got over $90 \%$ in the exam but here I'll get what under $60 \%$ and it sort of, try not to compare it but it does feel a bit like well $60 \%$ is a lot lower than $90 \%$.

(Student 4, Male, passed 12 stations, quartile rank 2, spent 21 minutes on the website)

\section{Disconnection between assessment and future learning}

There was a disconnection between clinical learning in the workplace and the OSCE. While spending extensive time seeing patients in the clinical workplace was seen as essential in order to gain a good mark in the OSCE, feedback from the OSCE was not seen as necessary for future learning in the workplace. It was seen as relevant only for that specific exam (or for a specific question within the exam) and the variability of their performance across stations made it irrelevant to consider their performance of generic skills across several stations. If there were plans to use the feedback in the future, this was in connection with future OSCEs, not workplace learning.

Erm, I know when it comes to OSCEs next year, I'll probably look at the website again and compare my marks, and think, 'Right, okay then, I didn't get as many marks on this bit. I need to work on that bit,"

(Student 11 Female, passed 11 stations, quartile rank 2, spent 16 minutes on the website) 
Feedback was rarely discussed with tutors in the workplace to encourage further consideration of the feedback after the emotional response to the release of the results had subsided. When it did take place, this was helpful.

My GP [tutor] asked me areas I could improve so.... looked at the OSCE thing to see which stations I could have improved on and we went through them, which was quite useful."

(Student 14, Male, passed 11 stations, quartile rank 1, spent 36 minutes on the website)

\section{DISCUSSION}

We aimed to explore why students failed to make more use of feedback after summative assessment, and which factors were influential. This study has demonstrated how the dominance of the summative assessment culture influences behaviour, emotions and cognitions regarding feedback. This fosters the reductionist approach to assessment, with the main aim to avoid failure. Students are very relieved to pass and, as they feel they have achieved their goal, they perceive little incentive to address their weaknesses. Motivation is known to be a critical factor in the response to feedback (Hattie and Timperley, 2007). Other studies have previously shown the strong connection between emotions and academic achievement, but the influence on feedback utilisation has not previously been considered in detail (Pekrun el al., 2002).The assessments are disconnected from most other clinical workplace learning in the minds of the students and this is reinforced by the attitudes of clinical teachers and family members. 
Our study has also demonstrated that students seek to manage the perceptions of others, particularly their parents, by using the feedback to demonstrate how well they are doing, or to show how difficult the course is. This has some similarities with the organisational psychology literature, in which employees seek feedback not to obtain information, but rather to highlight information to others (Ashford and Cummings, 1983; Morrison and Bies, 1991). We did not find clear evidence of active feedback-avoiding behaviour.

Although there appeared to be little evidence that students failed to understand the feedback message, there was confirmatory evidence that learners were often not motivated to make use of the feedback. This was partly because the students disconnected the OSCES from clinical workplace learning and partly because the summative culture encouraged a focus on avoiding failure. As such, they had achieved their goal, so students failed to perceive a 'feedback gap'.

The findings from this study reinforce the notion that learning from feedback cannot be dissociated from the context in which it takes place, as described by situativity theory (Durning and Artino, 2011). This theory proposes that educators need to consider not just the method by which information is given to learners, but instead focus on understanding the situations in which the information will, or will not, be used. The learning environment is as important as the way a learner internally processes information. The theory also proposes that authentic learning activities should result in better learning (Durning and Artino, 2011). The summative, high-stakes assessment culture, with its focus on passing and 
failing, with concomitant rewards and punishments, along with a disconnection from the authentic clinical workplace learning, is critical. Other important elements of this context include the social interactions with others, whether it be fellow students or the more hierarchical influence of teachers and parents. It is important to understand how this context can limit the transferability of learning following an assessment to the learning desired in the clinical workplace. At the same time, it is important to recognise that the way in which learners respond to feedback is not uniform. Furthermore, learners are actually not simply passive 'consumers' of the learning and assessment environment, but are generally 'agentic learners' who actively seek to influence their own learning and try to adapt the environment to be maximally conducive to their learning (Bandura, 2001). In this light the stifling effect that summative aspect has on the uptake and use of feedback is even more striking.

This study provides further insights into the complex effects that summative, high-stakes assessment has on receiving and learning from feedback. Our findings show some similarities to the pre-assessment effects of summative assessments (Rudland et al., 2008; Cilliers et al., 2010, 2012; O'Carroll and Fisher, 2013). In both cases there are complex and multifaceted interactions between various factors in an assessment system which together influence learning, sometimes in a positive way, but often in a negative manner. Students pick up implicit messages from the teaching staff and their fellow students regarding the expectations of the learning that is required. 


\section{Implications for Practice}

An appreciation of the relevance of socio-cultural theory to feedback in this context leads to some challenging conclusions. Asking faculty to provide more, or better-quality, feedback will have only limited success. Similarly, encouraging students to reflect on their results and their feedback is likely to have little impact. Instead, the focus should be on the development of a 'feedback culture' (Archer, 2010). Achieving this culture within the medical profession seems particularly problematic when compared with other professional cultures such as sports and music, as feedback is often given in a sporadic rather than planned way, and on the basis of inference rather than directly observed performance (Watling et al., 2013). Unlike medicine, music and sport both have a culture in which a tutor or coach has a more clearly defined and developed educational relationship with the learner, which facilitates the provision (and acceptance) of more challenging feedback. Nevertheless, the potential value of feedback requires that we face the challenge and consider potentially radical solutions.

This study's findings about the negative effects of the dominant summative assessment culture therefore support the calls to move away from a pure focus on assessment of learning and towards a more integrated approach which also incorporates assessment for learning (Schuwirth and van der Vleuten, 2011). One of the previous drivers for this has been a recognition that it is inappropriate to rely on a single assessment point, as this is inevitably flawed (van der Vleuten et al., 2012). To counter this, a programmatic approach to assessment was first proposed some years ago (van der Vleuten and Schuwirth, 2005). Since that time, quality criteria and guidelines for such programmes have been developed 
(Dijkstra et al., 2010). More recently a theory-driven framework for the practical implementation of a programmatic assessment model has been proposed (van der Vleuten et al., 2012). This model is designed for learner-centred programmes which favour deep learning strategies and is designed to have three main purposes: it should support assessment for learning; it should enable high-stakes decisions to be made using robust evidence; it should produce information that enables the curriculum to be improved. This model is theoretically coherent and there is emerging evidence that it can be practically implemented in different settings (Dannefer and Henson, 2007; Driessen et al., 2012). Initial evidence suggests it may well be beneficial in encouraging the development of selfregulated learning behaviour among learners and a feedback culture, though more evidence is needed (Altahawi et al., 2012).

However there are a number of challenges that threaten the practical implementation of this model. Medical schools are often restricted by university regulations or other legal restrictions which embed a summative assessment culture. Aside from this, there may be faculty resistance to whole-scale changes in the programme of assessment and in particular to the perceived downplaying of the importance of stand-alone summative assessment. This may occur despite evidence that summative assessment can have significantly adverse effects on students' learning (Cilliers et al., 2010, 2012).

\section{Limitations}


There are some limitations to our study. It was conducted in a single centre with a single year group and used a single summative assessment method. We do not know if participants would have given more or less attention to feedback if the assessment had not been summative. Interview studies are by their nature limited to considering only the participants' perspectives and reports of how they use the feedback. Only one interviewee was from the 'just passed' group and none had failed the OSCE. We have tried to enhance the credibility of the findings by using multiple data sources to triangulate the findings, linking the interview data with the way each participant used the website and performed in the assessment. The findings seem to resonate with much of the existing feedback literature based on formative assessment, as well as the literature about the preparation for written summative exams. Our context is not unusual - as most undergraduate courses have summative OSCEs - and is therefore applicable to many medical schools. This therefore suggests that the findings may be transferable.

\section{Suggestions for Further Research}

In this study we have investigated factors which affect medical students' utilisation of feedback in the context of summative assessment. Further research is required to investigate if these factors are replicated in other settings. Before considering abandoning the concept of summative assessment, we need to explore the acceptability, among both faculty and students, of changing to more programmatic form of assessment, in which the focus is on both assessment for learning and assessment of learning. It is also important to study students' engagement with feedback in medical schools which are implementing a programmatic approach to assessment where a feedback culture has been fostered. 


\section{CONCLUSIONS}

All assessments generate large amounts of data about individual students which can potentially inform and enhance future learning. However, the culture of summative assessment, with its focus on passing and failing, can act as a disincentive for use of this rich feedback information. Yet it is clear that a separation of summative and formative assessments at the programme level does not work; summative assessments do not provide meaningful feedback and purely formative assessment tends to be taken less seriously in the long run. For an assessment for learning programme therefore, the formative function and summative functions need to be combined in all assessments. Our study has shed light on the factors that have to be manipulated in the assessment programme in order to make this combination work.

\section{Acknowledgements}

We are grateful to Sarah Yardley for independently coding some of the interviews, to Adrian Molyneux for setting up the feedback website and to Kirsty Hartley for helping to recruit students for the interviews. Finally, we are most grateful to all the students who took part in the interviews.

\section{REFERENCES}


Altahawi, F., Sisk, B., Poloskey, S., Hicks, C. \& Dannefer, E. F. (2012). Student perspectives on assessment: experience in a competency-based portfolio system. Medical Teacher, 34, 221225

Archer, J.C. (2010). State of the science in health professional education: effective feedback. Medical Education, 44, 101-108

Ashford, S. J. \& Cummings, L. L. (1983). Feedback as an individual resource - personal strategies of creating information. Organizational Behavior \& Human Performance, 32, 37098

Bandura, A. (2001). Social cognitive theory. Annual Review of Psychology, 52, 1-26

Bok, H. G. J., Teunissen, P. W., Spruijt, A., Fokkema, J. P. I., van Beukelen, P., Jaarsma, D. A. D. C. \& van der Vleuten, C. P. M. (2013). Clarifying students' feedback-seeking behaviour in clinical clerkships. Medical Education, 47, 282-91

Cilliers, F. J., Schuwirth, L. W. \& van der Vleuten C. P. M. (2012). A model of the preassessment learning effects of assessment is operational in an undergraduate clinical context. BMC Medical Education, 12, 9.

Cilliers, F. J., Schuwirth, L. W., Adendorff, H. J., Herman, N. \& van der Vleuten, C. P. M. (2010). The mechanism of impact of summative assessment on medical students' learning. Advances in Health Sciences Education, 15, 695-715

Corbin, J. \& Strauss A. (2008). Basics of Qualitative Research, $3^{\text {rd }}$ edn. (Thousand Oaks, CA: Sage Publications) 
Crommelinck, M. \& Anseel, F. (2013). Understanding and encouraging feedback-seeking behaviour: a literature review. Medical Education, 47, 232-241

Dannefer, E. F. \& Henson, L. C. (2007). The portfolio approach to competency-based assessment at the Cleveland Clinic Lerner College of Medicine. Academic Medicine, 82, 493502

DiCicco-Bloom, B, \& Crabtree, B. F. (2006). The qualitative research interview. Medical Education, 40, 314-321

Dijkstra, J., van der Vleuten, C. P. M. \& Schuwirth, L. W. (2010). A new framework for designing programmes of assessment. Advances in Health Sciences Education, 15, 379-393

Dochy, F., Segers, M., Gijbels, D. \& Struyven, K. (2007). Assessment Engineering: Breaking down barriers between teaching and learning, and assessment. (In D. Boud \& N. Falchikov (Eds.), Rethinking assessment in higher education: learning for the longer term. (pp 87-100) Oxford: Routledge.)

Driessen, E. W., van Tartwijk, J., Govaerts, M., Teunissen, P. \& van der Vleuten, C. P. M. (2012). The use of programmatic assessment in the clinical workplace: a Maastricht case report. Medical Teacher, 34, 226-231

Durning, S. \& Artino, A. (2011). Situativity theory: a perspective on how participants and the environment can act. Medical Teacher, 33, 188-199

Eva, K. W., Armson, H., Holmboe, E., Lockyer, J., Loney, E., Mann, K., Sargeant, J. (2012). Factors influencing responsiveness to feedback: on the interplay between fear, confidence, and reasoning processes. Advances in Health Sciences Education 2012, 17, 15-26 
Harrison, C. J., Könings, K. D., Molyneux, A., Schuwirth, L., Wass, V. \& van der Vleuten, C. P. M. (2013). Web-based feedback after summative assessment: how do students engage? Medical Education, 47, 734-744

Hattie, J. \& Timperley, H. (2007). The power of feedback. Review of Educational Research, $77,81-112$

Kluger, A. N. \& DeNisi, A. (1996). The effects of feedback interventions on performance; a historical review, a meta-analysis and a preliminary feedback intervention theory. Psychological Bulletin, 119, 254-284

Lingard, L., Albert, M. \& Levinson, W. (2008). Grounded theory, mixed methods and action research. $B M J, 337,459-461$

Mann, K., van der Vleuten, C., Eva, K., Armson, H., Chesluk, B., Dornan, T., Holmboe, E., Lockyer, J., Loney, E., Sargeant, J. (2011). Tensions in informed self-assessment: how the desire for feedback and reticence to collect and use it can conflict. Academic Medicine, 86, $1120-1127$

Morrison, E. W. \& Bies, R.J. (1991). Impression management in the feedback-seeking process - a literature review and research agenda. Academy of Management Review, 16, $522-41$

Moss, S. E., Valenzi, E. R. \& Taggart, W. (2003). Are you hiding from your boss? The development of a taxonomy and instrument to assess the feedback management behaviours of good and bad performers. Journal of Management, 29, 487-510 
Norcini, J., Anerson, B., Bollela, V., Burch, V., Costa, M. J., Duvivier, R., Galbraith, R., Hays, R., Kent, A., Perrott, V. \& Roberts, T. (2011). Criteria for good assessment: Consensus statement and recommendations from the Ottawa 2010 Conference. Medical Teacher, 33, 206-214

Northcraft, G. B. \& Ashford, S. J. (1990). The preservation of self in everyday life - the effects of performance expectations and feedback context on feedback inquiry. Organizational Behavior \& Human Decision Processes, 47, 42-64

O'Carroll, P. J. \& Fisher, P. (2013). Metacognitions, worry and attentional control in predicting OSCE performance test anxiety. Medical Education, 47, 562-568

Patton, M. Q. (2002). Qualitative Research and Evaluation Methods, 3rd edn. (Newbury Park, CA: Sage Publications)

Pekrun, R., Goetz, T., Titz, W., \& Perry, R. P. (2002). Academic emotions in students' selfregulated learning and achievement: A program of qualitative and quantitative research. Educational psychologist, 37(2), 91-105.

Ramani, S. \& Krackov, S. K. (2012). Twelve tips for giving feedback effectively in the clinical environment. Medical Teacher, 34, 787-791

Rudland, J., Wilkinson, T., Smith-Han, K. \& Thompson-Fawcett, M. (2008). "You can do it late at night or in the morning. You can do it at home, I did it with my flatmate." The educational impact of an OSCE. Medical Teacher, 30, 206-211

Sargeant, J., Mann, K., Sinclair, D., van der Vleuten, C. \& Metsemakers, J. Understanding the influence of emotions and reflection upon multi-source feedback acceptance and use. Advances in Health Sciences Education, 13, 275-288 
Schuwirth, L. W. T. \& van der Vleuten, C. P. M. (2011). Programmatic assessment: from assessment of learning to assessment for learning. Medical Teacher, 33, 478-485

Shute, V. (2008). Focus on formative feedback. Review of Educational Research, 78, 153-89

Sinclair, H. K. \& Cleland, J. A. (2007). Undergraduate medical students: who seeks formative feedback? Medical Education, 41, 580-582

Teunissen, P. W., Stapel, D. A., van der Vleuten, C. P. M., Scherpbier, A. J. J. A., Boor, K. \& Scheele, F. (2009). Who wants feedback? An investigation of the variables influencing residents' feedback-seeking behavior in relation to night shifts. Academic Medicine, 84, 910917

Van de Ridder, J.M., Stokking, K.M., McGaghie, W.C, \& ten Cate, O. T. J. (2008). What is feedback in clinical education? Medical Education, 42, 189-197

van der Vleuten, C. P. M. \& Schuwirth, L. W. (2005). Assessment of professional competence: from methods to programmes. Medical Education, 39, 309-317

van der Vleuten, C. P. M., Schuwirth, L. W., Driessen, E., Dijkstra, J., Tigelaar, D., Baartman, L. K. J. \& van Tartwijk, J. (2012). A model for programmatic assessment fit for purpose. Medical Teacher, 34, 205-214

Watling, C., Driessen, E., van der Vleuten, C. P. M., Vanstone, M. \& Lingard, L. (2013). Beyond individualism: professional culture and its influence on feedback. Medical Education, 47, 585-594

Yardley, S., Teunissen, P. W. \& Dornan, T. (2012). Experiential learning: AMEE guide No. 63. Medical Teacher, 34, e100-e115 


\section{Page32}


Table 1: Summary of Participant Characteristics

\begin{tabular}{|l|l|l|l|l|l|l|l|l|l|l|l|l|l|l|l|l|l|l|l|l|}
\hline Student & 1 & 2 & 3 & 4 & 5 & 6 & 7 & 8 & 9 & 10 & 11 & 12 & 13 & 14 & 15 & 16 & 17 \\
\hline Gender & $\mathrm{F}$ & $\mathrm{M}$ & $\mathrm{F}$ & $\mathrm{M}$ & $\mathrm{M}$ & $\mathrm{M}$ & $\mathrm{F}$ & $\mathrm{F}$ & $\mathrm{F}$ & $\mathrm{F}$ & $\mathrm{F}$ & $\mathrm{F}$ & $\mathrm{M}$ & $\mathrm{M}$ & $\mathrm{M}$ & $\mathrm{F}$ & $\mathrm{F}$ \\
\hline School entrant or graduate entrant & $\mathrm{G}$ & $\mathrm{S}$ & $\mathrm{S}$ & $\mathrm{S}$ & $\mathrm{S}$ & $\mathrm{S}$ & $\mathrm{S}$ & $\mathrm{S}$ & $\mathrm{S}$ & $\mathrm{S}$ & & $\mathrm{S}$ & $\mathrm{S}$ & $\mathrm{S}$ & $\mathrm{G}$ & & $\mathrm{G}$ \\
\hline Total webpages viewed & 316 & 311 & 46 & 134 & 111 & 261 & 93 & 132 & 117 & 279 & 75 & 67 & 119 & 232 & 71 & 64 & 134 \\
\hline Total visits to website & 5 & 5 & 1 & 3 & 2 & 3 & 2 & 2 & 4 & 2 & 1 & 1 & 3 & 3 & 2 & 1 & 2 \\
\hline Pages viewed per minute & 4.9 & 4.9 & 7.7 & 6.4 & 13.9 & 5.0 & 9.3 & 9.4 & 1.7 & 8.0 & 4.7 & 5.6 & 7.4 & 6.4 & 11.8 & 10.7 & 8.9 \\
\hline Total time & 65 & 63 & 6 & 21 & 8 & 52 & 10 & 14 & 70 & 35 & 16 & 12 & 16 & 36 & 6 & 6 & 15 \\
\hline OSCE score as \% & 81 & 81 & 84 & 82 & 78 & 75 & 67 & 82 & 70 & 83 & 70 & 79 & 81 & 83 & 71 & 74 & 72 \\
\hline OSCE stations passed (out of 12) & 12 & 12 & 12 & 12 & 10 & 12 & 8 & 10 & 10 & 12 & 11 & 12 & 12 & 11 & 10 & 11 & 11 \\
\hline Quartile rank (based on all assessments) & 2 & 4 & 1 & 2 & 1 & 3 & 4 & 3 & 3 & 1 & 2 & 2 & 1 & 1 & 2 & 4 & 3 \\
\hline
\end{tabular}

Notes

$\mathrm{M}=$ male, $\mathrm{F}=$ female

$\mathrm{S}=$ entered medical school straight from school. $\mathrm{G}=$ entered medical school as graduate (two students declined to provide this information)

Quartile rank represents rank in year based on all assessments: 1 is top quartile etc 